\section{Simultaneous repair of cardiac pathology and severe pectus excavatum in Marfan patients using a modified minimally invasive repair}

\author{
Dawn E. Jaroszewski, Jason D. Fraser, \\ Patrick A. DeValeria \\ Cardiothoracic Surgery, Mayo Clinic \\ Arizona, Phoenix, USA
}

\begin{abstract}
Pectus excavatum (PE) deformity is present in the majority of Marfan patients. Many have not had PE repair and present as adults with aortic and valve pathology requiring operative intervention. We present our preliminary report of simultaneous cardiac surgery and repair of the chest wall deformity. Utilizing this modified minimally invasive excavatum repair provided a quick, safe repair with good cosmetic results.
\end{abstract}

\section{Introduction}

Marfan syndrome is an autosomal dominant connective tissue disorder affecting the skeletal, ocular, cardiovascular and pulmonary systems. ${ }^{1-3}$ The skeletal system is most commonly involved and a severe pectus excavatum (PE) deformity is present in a large majority of patients with Marfan syndrome.,3 Marfan patients often have cardiovascular involvement including aortic and valve pathology. ${ }^{1-3}$ Adult patients presenting with cardiac disease may also have a severe PE deformity (Figure 1). Repair of both the PE and cardiac disease in a single stage is preferable to avoid postoperative difficulties that may result from cardiac compression or displacement into the left thoracic cavity. ${ }^{4-6}$ Historically, modifications of the open Ravitch-type procedure have been used exclusively for simultaneous repair of the PE and cardiac disease due to the complex nature and greater severity of deformities in adult patients with Marfan syndrome.,4,6 Since its introduction by Nuss et al., ${ }^{8}$ clinical results have shown the Minimally Invasive Repair PE (MIRPE) to be safe and successful in the Marfan syndrome patient.-10 We describe simultaneous cardiac surgery and chest wall repair in two Marfan adults using a modified MIRPE. Good surgical exposure, excellent sternal stability and satisfactory cosmetic appearances were obtained.

\section{Case Report}

\section{Patient \#1}

A 22-year-old man with pectus index of 9.2, diagnosed and closely followed since age 6 with de novo Marfan syndrome (skeletal features, dilation of the aorta at the level of the sinuses of the Valsalva, ectopia lentis, and significant skeletal findings including scoliosis, pectus excavatum and wrist, thumb, and elbow joint hypermobility, high arched palate with tooth crowding, characteristic facial appearance), underwent ascending aortic root replacement with Gelweave ${ }^{\mathrm{TM}} 28 \mathrm{~mm}$ graft (Vascutek Ltd., Scotland, UK), reimplantation of coronaries and $21 \mathrm{~mm}$ mechanical aortic valve replacement (St. Jude Medical Inc., Minneapolis, MN, USA) with subsequent PE repair. Operating time was 108 minuntes and hospitalization was 8 days. A left pleural effusion requiring thoracentesis was the only morbidity.

\section{Patient \#2}

A 52-year-old man with pectus index of 4.5, diagnosed and closely followed since his early 40's with de novo Marfan syndrome (skeletal features including scoliosis, pectus excavatum, joint hypermobility, mitral valve prolapse syndrome, myopia, and borderline non-progressive aortic enlargement), underwent mitral valve repair including posterior leaflet sliding repair, anterior leaflet plication, chordal shortening, annuloplasty with $34 \mathrm{~mm}$ band (Edwards Lifesciences, Irvine, CA, USA) and subsequent $\mathrm{PE}$ repair. Operating time was 92 minuntes and hospitalization was 6 days. Atrial fibrillation complicated the postoperative course.

\section{Procedure details}

Supine positioning with arms tucked and standard median sternotomy incision were used. Due to the severity of the deformities, extensive dissection and upward traction with rake retractors on the lower sternal margin was performed during sternotomy to prevent damage to underlying compressed right ventricle and atrium. Bicaval cannulation, heparinization and cardiopulmonary bypass were utilized to perform the cardiac procedures previously stated. After separation from bypass, protamine was administered and hemostasis obtained. In order to protect and prevent adhesions of the support bars to the heart, pericardial reconstruction was performed. CorMatrix ${ }^{\circledR}$ pericardial closure patch (CorMatrix $^{\circledR}$ Cardiovascular Inc., Sunnyvale, CA, USA) was used to close the pericardium for patien \#1. Chest tubes were placed in the mediastinum and pleural cavities.

Repair of the PE defect was then carried out. The most severe portion of the defect had been
Correspondence: Dawn Jaroszewski, Division of Cardiothoracic Surgery, Mayo Clinic Arizona, 5777 E. Mayo Boulevard, Phoenix, AZ 85054, USA. Tel. +1.480.342.2270 - Fax: +1.480.342.2269.

E-mail: Jaroszewski.dawn@mayo.edu

Key words: Pectus excavatum, aortic aneurysm, valve replacement, minimally invasive pectus repair, Nuss, Marfan's syndrome.

Received for publication: 7 March 2011. Accepted for publication: 29 April 2011.

This work is licensed under a Creative Commons Attribution NonCommercial 3.0 License (CC BYNC 3.0).

@C Copyright D.E. Jaroszewski et al., 2011

Licensee PAGEPress, Italy

Chest Disease Reports 2011; 1:e3

doi:10.4081/cdr.2011.e3

marked out preoperatively on the skin and the intercostal spaces identified bilaterally for placement of support bars. Because of the significant sternal deformity, it was necessary to place several single sternal wires to partially approximate the sternum to achieve correct alignment when placing the bars. Both patients required 2 bars to achieve full correction. Bilateral anterior axillary incisions were made centered between the intercostal spaces identified for bar placement. ${ }^{8-10}$ The pectoralis major muscle was dissected from the chest wall bilaterally and the thoracic entry and exit sites in the intercostal sites made close to the sternum with a large introducer (Lorenz

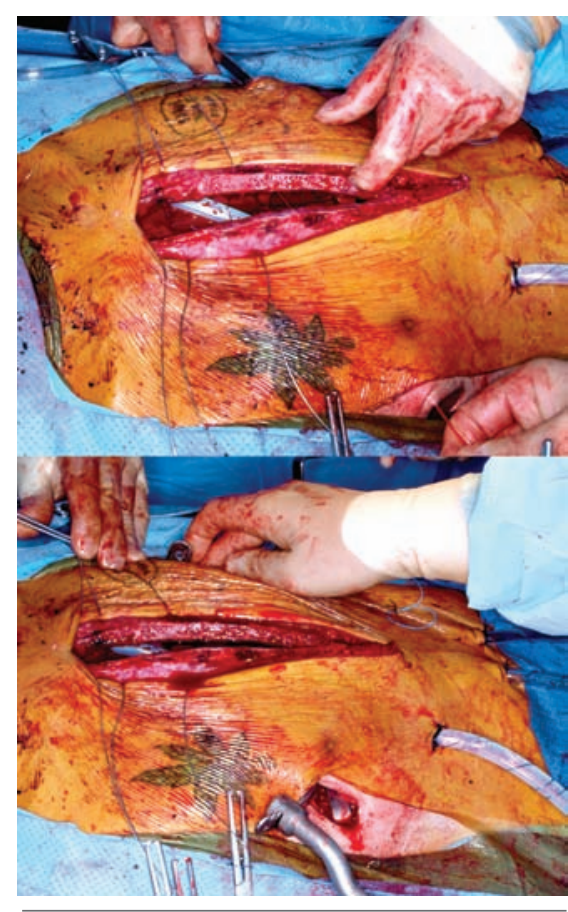

Figure 1. Placement of the substernal bar under direct vision and flipping of the bar. 
Surgical, Jacksonville, FL, USA). Because the sternum was still not completely approximated, full visualization and safe guidance of the introducer across the defect could be performed without thoracoscopy. The sternal wires were tightened to approximate the sternum and the introducer lifted anterior to pull the sternum and chest wall out of the depressed position. This was repeated several times to loosen the anterior chest wall. An umbilical tape was secured to the end of the introducer and then left as a guide after removal of the introducer. The sternal wires were loosened to again allow several centimeters of visualization through the sternotomy. The umbilical tape was secured to the first bar and the bar guided through the intercostal thoracic entrance site and back through the contralateral intercostal exit site (Figure 1A). A second bar was placed in a similar fashion. Both bars were then flipped $180^{\circ}$ and the sternum pulled anterior (Figure 1B). The sternal edges were no longer flush after correction of the PE and remodeling was necessary to allow precise approximation and stable sternal closure. With a mallet and osteotome wedge, an even parallel surface was carved allowing close approximation of the sternum, which was then wired together. The remaining wires (total of 8 ) were placed and the sternum closed.

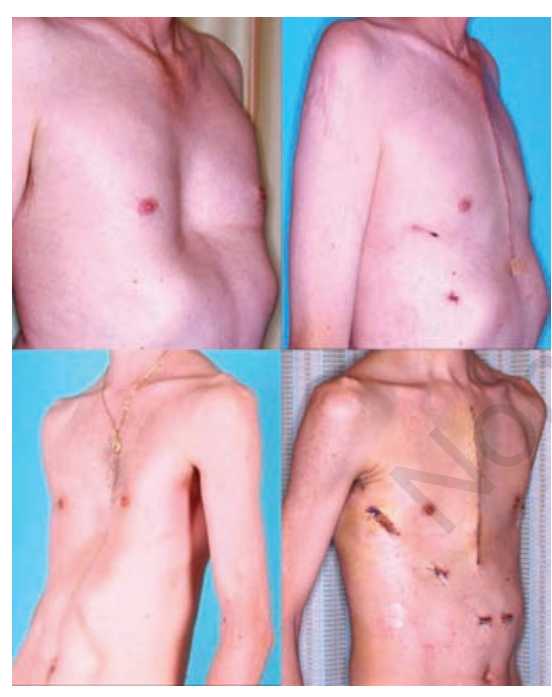

Figure 2. Patients \#1 and \#2 pre-and one week post-operative cardiac surgery and simultaneous minimally invasive repair of pectus excavatum deformity.
Stabilizers (Lorenz Surgical) were placed on one side of each bar and attached to the ribs. The muscle and subcutaneous tissues were closed over the bars and stabilizers. The skin was closed and dressings applied. Post operative pain was managed with patient controlled anesthesia versus our standard of thoracic epidural due to heparin utilization during cardiac bypass. Preliminary results and follow-up on both patients are now greater than 28 months with no discomfort and excellent cosmetic results (Figure 2). Bar removal is planned at 36 months post-operative.

\section{Discussion}

A severe PE deformity is present in up to $75 \%$ of Marfan patients. ${ }^{3,10}$ Many have not had PE repair and present as adults with aortic and valve pathology requiring operative intervention. ${ }^{5,10}$ Simultaneous cardiac surgery and repair of the chest wall deformity can be safely performed. Our modification of the MIRPE included direct visualization when passing the introducer and bars across the thoracic cavity through a partially approximated median sternotomy. This allowed for safe and controlled passage of the introducer and bars. A similar technique was recently described as a single case report in non-Marfan patients with good outcomes. ${ }^{9}$

Lateral bar stabilizers were used as recommended by Nuss, et al..$^{8,10}$ In these patients, the bulk of the stabilizers created discomfort requiring removal after several months and it is our practice to no longer utilize stabilizers. Bars are directly affixed to the chest wall with no. 5 FiberWire $^{\circledR}$ (Arthrex Inc., Naples, FL, USA) incorporating both the bar and rib at several fixation sites bilateral.

\section{Conclusions}

Simultaneous cardiac surgery and repair of PE is feasible and should be considered in the Marfan syndrome patient. Despite a more severe defect, the MIRPE procedure has been shown to provide long-term repair of PE in the Marfan patient and can be used successfully during concurrent cardiac surgery. ${ }^{10}$

\section{References}

1. De Paepe A, Devereux RB, Dietz HC, et al. Revised diagnostic criteria for the Marfan syndrome. Amer J Med Genet 1996;62:41726.

2. Loeys BL, Dietz HC, Braverman AC, et al. The revised Ghent nosology for the Marfan syndrome. J Med Genet 2010;47:476-85.

3. Pyeritz RE, McKusick VA. The Marfan syndrome: diagnosis and management. $\mathrm{N}$ Engl J Med 1979;300:772-7.

4. Hasegawa T, Yamaguchi M, Ohshima Y, et al. Simultaneous repair of pectus excavatum and congenital heart disease over the past 30 years. Eur J Cardiothorac Surg 2002;22:874-8.

5. Miller DR, Pugh DM. Repair of ascending aortic aneurysm and aortic regurgitation complicated by acute cardiac compression by pectus excavatum in Marfan's syndrome. J Thorac Cardiovasc Surg 1970;59: 673-84.

6. Okay T, Ketenci B, Imamoglu OU, et al. Simultaneous open-heart surgery and pectus deformity correction. Surg Today 2008;38:592-6.

7. Karl TR. A technique for concurrent repair of pectus excavatum and intracardiac defects. J Card Surg 1988;3:487-9.

8. Nuss D. Minimally invasive repair of pectus excavatum. Semin Pediatr Surg 2008;17:209-17.

9. Kao CH, Tsai CS, Huang TW, et al. The Nuss procedure for pectus excavatum at the time of atrial septal closure. Ann Thorac Surg 2010;89:985-6.

10. Redlinger RE Jr, Rushing GD, Moskowitz $\mathrm{AD}$, et al. Minimally invasive repair of pectus excavatum in patients with Marfan syndrome and marfanoid features. J Pediatr Surg 2010;45:193-9. 